\title{
Ion-specific thermodynamical properties of aqueous proteins
}

\author{
EDUARDO R.A. LIMA ${ }^{1,2}$, EVARISTO C. BISCAIA Jr. ${ }^{2}$, \\ MATHIAS BOSTRÖM $M^{3,4}$ and FREDERICO W. TAVARES ${ }^{1}$ \\ ${ }^{1}$ Escola de Química, Universidade Federal do Rio de Janeiro, \\ Centro de Tecnologia, Bloco E, sala 207, Cidade Universitária, 21949-900 Rio de Janeiro, RJ, Brasil \\ ${ }^{2}$ Programa de Engenharia Química, COPPE, Universidade Federal do Rio de Janeiro, \\ Centro de Tecnologia, Bloco G, Sala 116, Cidade Universitária, 21941-972 Rio de Janeiro, RJ, Brasil \\ ${ }^{3}$ Institute of Physical and Theoretical Chemistry, University of Regensburg, D-93040 Regensburg, Germany \\ ${ }^{4}$ Division of Theory and Modeling, Department of Physics, Chemistry and Biology, \\ Linköping University, SE-581 83 Linköping, Sweden
}

Manuscript received on April 28, 2008; accepted for publication on May 27, 2009

\begin{abstract}
Ion-specific interactions between two colloidal particles are calculated using a modified Poisson-Boltzmann (PB) equation and Monte Carlo (MC) simulations. PB equations present good results of ionic concentration profiles around a macroion, especially for salt solutions containing monovalent ions. These equations include not only electrostatic interactions, but also dispersion potentials originated from polarizabilities of ions and proteins. This enables us to predict ion-specific properties of colloidal systems. We compared results obtained from the modified $\mathrm{PB}$ equation with those from $\mathrm{MC}$ simulations and integral equations. Phase diagrams and osmotic second virial coefficients are also presented for different salt solutions at different $\mathrm{pH}$ and ionic strengths, in agreement with the experimental results observed Hofmeister effects. In order to include the water structure and hydration effect, we have used an effective interaction obtained from molecular dynamics of each ion and a hydrophobic surface combined with PB equation. The method has been proved to be efficient and suitable for describing phenomena where the water structure close to the interface plays an essential role. Important thermodynamic properties related to protein aggregation, essential in biotechnology and pharmaceutical industries, can be obtained from the method shown here.
\end{abstract}

Key words: colloid stability, Hofmeister series, potential of mean force, proteins.

\section{INTRODUCTION}

In a series of papers in the late 1880's, Franz Hofmeister could demonstrate that salts with fixed cations and different anions have different capacities in stabilizing protein suspensions (Ninham and Yaminsky 1997, Kunz et al. 2004b). One of these experiments was on a dispersion of proteins of whole hen-eggwhite. Depending on the anion, different concentrations were required to precipitate a prescribed protein

Selected paper presented at the IUTAM Symposium on Swelling and Shrinking of Porous Materials: From Colloid Science to Poromechanics - August 06-10 2007, LNCC/MCT.

Correspondence to: Frederico W. Tavares

E-mail: tavares@eq.ufrj.br 
concentration. The salts could be ordered in a sequence of effectiveness that later seemed to be universal, being the same for a number of colloidal systems. Hofmeister's specific ion effects turn up frequently in biology and solution chemistry. For a long time the explanation for these effects has remained a mystery. Ries-Kautt and Ducruix (1989) investigated the relative effectiveness of various ions on the solubility of hen-egg-white lysozyme at $\mathrm{pH}$ 4.5. The experiments revealed that the effectiveness followed a reversed Hofmeister series: $\mathrm{SCN}^{-}>\mathrm{NO}_{3}^{-}>\mathrm{Cl}^{-}$. This was interesting since the original paper of Hofmeister (Kunz et al. 2004a) gives exactly the opposite anion sequence. This we term a direct Hofmeister series. Later on, the same group demonstrated a direct order for a protein studied at a $\mathrm{pH}$ higher than its $\mathrm{pI}$ (Carbonnaux et al. 1995).

In general, Hofmeister effects refer to the relative effectiveness of either anions or cations, individually or as ion pairs, on a wide range of phenomena. These effects have, for instance, been observed in experiments as diverse as double layer force measurements (Pashley and Ninham 1987, Boström et al. 2001a, Tavares et al. 2004a, b), bubble fusion (Craig et al. 1993), conformational changes of rhodopsin (Vogel 2004), bacterial cell growth (Lo Nostro et al. 2004), yield stresses in silica suspensions (Franks 2002), cutting-efficiency of DNA by restriction enzymes (Kim et al. 2001), charge of globular proteins (Curtis et al. 2002), surface tension of electrolytes (Boström et al. 2001b, 2005a), and the solubility of protein solutions (Kunz et al. 2004a, b, Ries-Kautt and Ducruix 1989, Carbonnaux et al. 1995).

The only ionic characteristics included in textbook descriptions of aqueous proteins in salt solutions are bulk $\mathrm{pH}$, salt concentration, ionic charges and "hydrated ionic radii". Electrostatic theories based on these parameters do not account for the experimentally observed ion specificity. Since standard theory failed, it has been customary to invoke various specific "ionic characteristics" such as: "structure breaking"; "structure creating"; "lyotropic"; "kosmotropic"; "chaotropic"; "salting-in"; and "salting-out" (Collins and Washabaugh 1985, Collins 2004). These concepts attempt to encompass the missing ion solvent interactions qualitatively. But the concepts have turned out to be difficult to quantify. There has been no quantitative explanation of the origin of the Hofmeister series.

It has only recently become evident that the non-electrostatic (NES) forces (Ninham and Yaminsky 1997, Kunz et al. 2004b), among which are those due to the ion specific polarizability of ions, neglected in previous theories, play an essential role in determining molecular forces above and beyond electrostatics, which is non-specific. Polarizability is a quantity that measures the response of a particle to a perturbation in the electromagnetic field. While two charged particles can interact via electrostatic forces, there is also a quantum mechanical attraction, the dispersion force, between two charge neutral particles that is related to the polarizabilities of the two particles. The same force is important also for ions. Dispersion forces have usually been assumed to be small compared to electrostatic forces, or when they have been considered and dismissed they were included incorrectly, or not at the same level as the other forces. The extensions of the Lifshitz theory do formally include ion specific hydration or self-energy, and hydration interactions (Boström and Ninham 2004, 2005). While electrostatic forces often dominate at low salt concentrations, NES forces become important at high salt concentrations where the electrostatic forces are weakened due to screening. We have shown in a series of papers that dispersion forces between an ion and an interface often dominate over electrostatic forces above and around physiological salt concentrations (Ninham and Yaminsky 1997, Boström et al. 2001a, b, 2005a, Tavares et al. 2004a, b).

Important recent contributions are addressed here, highlighting some novel techniques that give rise to 
good results when compared with molecular simulations and experiments. More specifically, we show the importance of considering the non-electrostatic interactions between ions and surfaces when modeling the interactions among proteins by Monte Carlo simulations in order to predict ion specific effects; we also show that a computationally efficient modified version of the Poisson-Boltzmann equation including these nonelectrostatic interactions gives good results for monovalent counterions when compared to more complex and time consuming methods, such as molecular simulations and integral equations. We show calculations of the reversal Hofmeister series, osmotic second virial coefficient, and phase diagram for globular protein solutions. In the second part of the paper, we used the PB equation with a much more realistic potential of mean force between each ion and the colloid interface and, in addition, we treated the dielectric constant as an inhomogeneous function close to the water-interface, both of them extracted from MD simulations. Using this approach, it is possible to capture the important physics of the system due to the inclusion of ion-surface van der Waals forces, short range hydration, image potential and different solvent-mediated forces. Then, we present ion specificity/competition in a mixture of salts near an uncharged nanoparticle. We show that soft ions are more attracted by hydrophobic surfaces. Of course, our calculation is done on a primitive model level, but nevertheless it is in reasonable agreement with recent results from molecular simulation and with experimental data from the literature.

Our program here is to use these NES forces to explain the ion specificity observed in protein solutions. Our main objective is to analyze the interaction forces between proteins and between other colloidal particles. To do so, we develop the necessary theoretical foundations for using Monte Carlo (MC) simulations to calculate the potential of mean force (PMF) between two colloidal particles in Section 2 (Tavares et al. 2004a). We also discuss how simple estimates for the NES forces can be obtained. The theory is then used in Section 3 to evaluate the ion specific PMF between two colloidal particles. In Section 4 we compare the ion distributions near a globular protein using Modified-Poisson-Boltzmann equation (MPB equation), hypernetted chain approximation, and solvent averaged MC simulations. This will demonstrate that, to a good approximation, one can often use MPB equation that includes ionic NES potentials to calculate ion distributions near globular proteins (Boström et al. 2005b, 2006). In Section 5 we present the calculation of the pressure between two planar surfaces coated with lysozyme proteins. We will demonstrate that, for $\mathrm{pH}<\mathrm{pI}$ (where the anions are counterions), the double layer repulsion increases in the order $\mathrm{NaSCN}<\mathrm{NaI}<\mathrm{NaCl}$. At higher $\mathrm{pH}>\mathrm{pI}$ (where the anions are coions), the double layer repulsion increases in the order $\mathrm{NaCl}<\mathrm{NaI}<\mathrm{NaSCN}$. This is in good agreement with both solubility experiments and second virial coefficients deduced from experiments using SAXS (Boström et al. 2005c). In fact, for large polarizable anions, such as thiocyanate, the double layer pressure can even become attractive at small protein separations. The MPB equation in bispherical coordinates discussed in Section 6 opens up for computer efficient calculations of protein-protein potentials of mean force. Then, in Section 7, we discuss different levels of approximations for the ion-protein potential of mean force. Recent work by Tavares and co-workers have demonstrated that the potential of mean force acting between each ion and a hydrophobic colloidal particle obtained from simulations that include explicit water molecules may be an important improvement compared to NES potentials from Lifshitz theory (Lima et al. 2007a, 2008a, b, Horinek et al. 2008). We finally present a short summary in Section 8 . Our results taken together provide a strong hint that we are close to the quantitative understanding of an important long standing question in solution chemistry and biology. 


\section{ION SPECIFIC MONTE CARLO SIMULATIONS AND NES POTENTIALS}

The potential of mean force (PMF) for two macroions is affected not only by the size and charge of each electrolyte ion, but also by the ion's polarizability (Tavares et al. 2004a). The Lifshitz theory provides a basis for calculating the van der Waals interaction between cation-colloid, anion-colloid, cation-cation and anion-anion pairs. Monte Carlo simulations can be used to determine how salt identity affects the PMF among colloidal particles or globular proteins in a saline solution.

Tavares et al. (2004a) focused on the contribution of ion-ion and ion-macroion van der Waals interactions to the colloid-colloid (or protein-protein) PMF. A primitive model, where water is represented by a dielectric continuum, was used.

The interaction energy between particles $i$ and $j$ is the sum of three parts:

$$
w_{i j}=w_{i j}^{h s}+w_{i j}^{e l e c}+w_{i j}^{d i s p} .
$$

The hard-sphere potential $w_{i j}^{h s}$ is

$$
w_{i j}^{h s}= \begin{cases}\infty & \text { for } r_{i j}<\left(\sigma_{i}+\sigma_{j}\right) / 2 \\ 0 & \text { for } r_{i j} \geq\left(\sigma_{i}+\sigma_{j}\right) / 2,\end{cases}
$$

where $r_{i j}$ is the center-to-center distance between particles $i$ and $j$ (ion or macroion). The electrostatic interaction $w_{i j}^{\text {elec }}$ is

$$
w_{i j}^{e l e c}=\frac{q_{i} q_{j}}{4 \pi \varepsilon_{0} \varepsilon r_{i j}} \quad \text { for } \quad r_{i j} \geq\left(\sigma_{i}+\sigma_{j}\right) / 2
$$

where $q$ and $\sigma$ are the charge and the diameter, respectively, of particle $i$ (ion or macroion); $\varepsilon_{0}=8.854 \times$ $10^{-12} \mathrm{C}^{2} /(\mathrm{Jm})$ is the dielectric permittivity of vacuum; and $\varepsilon$ is the dielectric constant of the solvent.

To calculate ion-ion dispersion interactions, we use Lifshitz theory (Israelachvili 1992) for van der Waals forces. The dispersion potential $w_{12}^{\text {disp }}$ between two small particles 1 and 2 (in this case, ions) in a medium 3 (the solvent) is given by:

$$
w_{12}^{d i s p}=\frac{-B_{12}}{r_{12}^{6}} \quad \text { for } \quad r_{12} \geq\left(\sigma_{1}+\sigma_{2}\right) / 2 .
$$

The dispersion parameter $B_{12}$ is calculated using multiple absorption frequencies (Israelachvili 1992),

$$
\frac{B_{12}}{k_{B} T}=3 \frac{\alpha_{1}(0) \alpha_{2}(0)}{\left[\varepsilon_{3}(0)\right]^{2}}+\frac{3 h}{\pi k_{B} T} \int_{\nu_{\min }}^{\infty} \frac{\alpha_{1}(v) \alpha_{2}(v)}{\left[\varepsilon_{3}(v)\right]^{2}} d v
$$

where $\alpha_{i}(0)$ and $\alpha_{i}(\nu)$ are the effective polarizabilities in water of particle $i$ at frequencies 0 and $\nu$, respectively; $v_{\min }=\frac{2 \pi k_{B} T}{h}$ is the first non-zero frequency; $h v_{\min }=2.59 \times 10^{13} \mathrm{erg}$ at $298 \mathrm{~K} ; v_{3}^{I}$ is the ionization frequency of the solvent, and $h v_{3}^{I}=20 \times 10^{-12} \mathrm{erg}$ is the ionization energy of the solvent (water); $h$ is Planck's constant; $\varepsilon_{3}(v)$ is the dielectric constant of the solvent at frequency $v$, and $\varepsilon_{3}(0)$ is the static dielectric constant, i.e., the dielectric constant of the solvent (for water $\varepsilon_{3}(0)=\varepsilon=78$, at $298 \mathrm{~K}$ ).

To calculate ion-colloid dispersion interactions, we note that the ion is much smaller than the macroion; therefore, the ion-macroion van der Waals interaction is approximated by the potential between a small spherical particle $i$ and a planar macroion $m$ (Israelachvili 1992):

$$
w_{i m}^{\text {disp }}=\frac{-B_{i m}}{d_{i m}^{3}} \quad \text { for } \quad d_{i m} \geq \sigma_{i} / 2,
$$


where $d_{i m}$ is the perpendicular distance from the center of the small particle $i$ (ion) to the surface of the macroion (colloid) $m$. The dispersion interaction parameter $B_{i m}$ is calculated from the Lifshitz theory (Ninham and Yaminsky 1997):

$$
\frac{B_{i m}}{k_{B} T}=\frac{\alpha_{i}(0)}{4 \varepsilon_{3}(0)}\left(\frac{\varepsilon_{m}(0)-\varepsilon_{3}(0)}{\varepsilon_{3}(0)+\varepsilon_{3}(0)}\right)+\frac{h}{4 \pi k_{B} T} \int_{v_{\min }}^{\infty} \frac{\alpha_{i}(v)}{\varepsilon_{3}(v)}\left(\frac{\varepsilon_{m}(v)-\varepsilon_{3}(v)}{\varepsilon_{m}(v)+\varepsilon_{3}(v)}\right) d v,
$$

where $\varepsilon_{m}(0)$ and $\varepsilon_{m}(\nu)$ are the dielectric constants of the macroion at frequencies 0 and $\nu$, respectively.

To calculate dispersion interaction parameters, $B_{i j}$ and $B_{i m}$, we need to know how the effective polarizabilities of ions and the dielectric constants for the macroion and the solvent vary with frequency. For a molecule with one characteristic absorption frequency (the ionization frequency), its polarizability and its dielectric constant at a frequency $v^{I}$ are given by the harmonic-oscillator model (Israelachvili 1992, Von Hipper 1958):

$$
\alpha(v)=\frac{\alpha(0)}{\left(1+\left(v / v^{I}\right)^{2}\right)}
$$

and

$$
\varepsilon(v)=1+\frac{\left(n^{2}-1\right)}{\left(1+\left(v / v^{I}\right)^{2}\right)},
$$

where $n$ is the refractive index for the sodium D line at $298 \mathrm{~K}$. Eq. (9) is used for both the solvent and for the macroion. Ninham and Parsegian (1970a, b) demonstrated that Eq. (8) can be used for atoms, and Ninham and Yaminsky (1997) later showed that it is also applied to cations and anions.

Eqs. (8) and (9) are substituted into Eqs. (5) and (7) to calculate the dispersion interactions between ion-ion and ion-macroion, respectively. Static dielectric constants, ionization energies and refractive indices for water and for colloid (or protein) are taken from Israelachvili (1992) and Tavares et al. (2004a). For cations and anions, the effective polarizabilities are given in Wu et al. $(1998,1999)$ and estimated ionization energies in aqueous solution in Tavares et al. (2004a).

The dispersion interaction parameters are obtained by integrating Eqs. (5) and (7) numerically. The resulting dispersion interactions at contact for ion-ion, Eq. (4) and ion-macroion, Eq. (6) are presented by Tavares et al. (2004a).

Canonical Monte Carlo simulation is used to calculate the mean force $F(r)$ between two macroions in electrolyte solution at a given center-to-center distance $r$. Potential of mean force $W$ is obtained by the integration of the mean force: $W(r)=\int_{\infty}^{r} F(r) d r$. Details of calculating the PMF from the mean force are given elsewhere (Wu et al. 1998, 1999).

In the Monte Carlo simulations, dispersion interactions of ion-ion and ion-macroion pairs are taken into account. The mean force between two macroion particles surrounded by small ions is the sum of four contributions (Tavares et al. 2004a),

$$
F(r)=-\frac{\partial w_{m m}^{e l e c}}{\partial r}\left\langle\sum_{i=1}^{N} \frac{\partial w_{i m}^{e l e c}}{\partial r}\right\rangle-\left\langle\sum_{i=1}^{N} \frac{\partial w_{i m}^{\text {disp }}}{\partial r}\right\rangle+F^{C}(r),
$$

where the angular brackets denote canonical ensemble average; $r$ is the center-to-center separation between two macroions; and $r_{i m}$ is the center-to-center distance between a small ion and a macroion. Subscripted $m$ denotes a macroion; superscripted elec denotes electrostatic (Coulomb) forces, and superscripted disp 
denotes van der Waals dispersion forces. The first term on the right hand side of Eq. (10) is the direct Coulomb interaction between two macroions; this term is always repulsive for like-charged macroions. In Eq. (10), the term corresponding to the direct dispersion interaction (Hamaker forces) between two macroions is set equal to zero. The Hamaker term is independent of the other contributions and can be included or modified after the calculation of all other terms is completed. The second term accounts for the Coulombic forces exerted on either of the macroions by all small ions. The third term accounts for the dispersion force exerted on either macroions by all small ions. The last term represents the mean force resulted from collisions between hard-sphere macroion particles and ions. The collision contribution to the mean force is calculated using (Tavares et al. 2004a, Wu et al. 1998):

$$
F^{C}(r)=-k_{B} T \lim _{\Delta r \rightarrow 0^{+}} \frac{\left\langle N_{C}\right\rangle}{\Delta r}-k_{B} T \lim _{\Delta r \rightarrow 0^{-}} \frac{\left\langle N_{C}\right\rangle}{\Delta r} .
$$

Here, $N_{C}$ is the number of salt ions that collide with a macroion due to a small range of distance $\Delta_{r}$ between the two macroions. Eq. (11) shows that the hard-sphere collision force is repulsive when small ions accumulate between the two macroions, and is attractive when they are depleted.

Monte Carlo simulation details are reported elsewhere (Tavares et al. 2004a, Wu et al. 1998, 1999).

\section{ION SPECIFIC PROTEIN-PROTEIN POTENTIAL OF MEAN FORCE}

In this section, we show some numerical results obtained using ion specific Monte Carlo simulations (Tavares et al. 2004a).

Figure 1 shows the simulation results for the total mean force $(F)$ between two positively charged macroions, with a diameter of $\sigma_{m}=20 \AA$ and a valence of +20 , in electrolyte solutions of $\mathrm{NaI}$ and $\mathrm{Na}_{2} \mathrm{SO}_{4}$ at ionic strength of $0.125 \mathrm{M}$. For all simulations, we use around 200 small ions, all with the same diameter $\sigma_{i}=4 \AA$, such that we have electroneutrality in the simulation box. The direct van der Waals interaction between two macroions (Hamaker) is not included. The mean forces are normalized using the Bjerrum length, $l_{B}=e^{2} /\left(4 \pi \varepsilon_{0} \varepsilon k_{B} T\right)$, that represents the distance between two unit charges $(e)$ where the pair potential equals thermal energy $k_{B} T$. Here, $T=298 \mathrm{~K}$ and $l_{B}=7.14 \AA$ (aqueous solutions). The line is the normalized mean force calculated using DLVO theory (in this case, only the double layer repulsion). As expected, the DLVO theory predicts repulsion for all intermacroion distances and overestimates this repulsion even for monovalent counterions. DLVO theory cannot represent the intercolloidal attraction that appears in the presence of divalent counterion.

The attractive dispersion forces between the counterion (iodide or sulfate) and the macroion increase the concentration of counterions around the macroion particles and, thereby, enhance both hard-sphere repulsion and screening of the electrostatic forces. The electrostatic force decays quickly with the distance and becomes insignificant at a separation distance sufficient to accommodate a monolayer of counterions in-between the two macroions. At about the same distance, we also observe rapid changes in the van der Waals forces and collision contributions to the mean force; these rapid changes are related to ion binding (Tavares et al. 2004a). This effect is stronger for sulfate (divalent) than iodide (monovalent). Bound counterions screen the electrostatic repulsion and create a bridge of attraction between two macroions. Therefore, the calculated total force can be attractive at short distances and the minimum is shifted to a shorter distance, as shown in Figure 1. Even though the structural solvation effects are neglected in these 


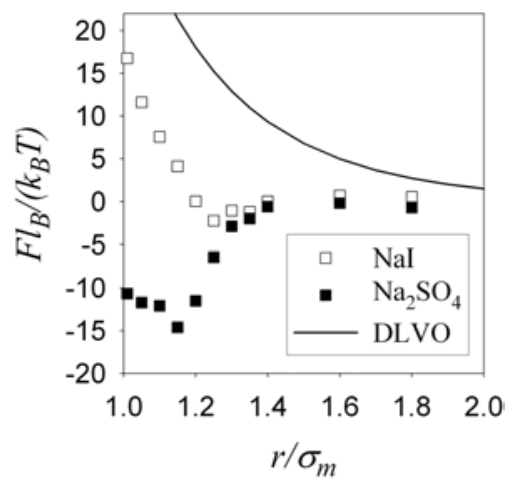

Fig. 1 - Total mean force between two macroions in monovalent and divalent electrolytes solution of ionic strength $0.125 \mathrm{M}$. Results are for $\mathrm{NaI}, \mathrm{Na}_{2} \mathrm{SO}_{4}$, and classical DLVO theory (Tavares et al. 2004b).

simulations, results shown in Figure 1 are consistent with the industrial practice where ammonium sulfate is commonly used to induce protein precipitation.

To illustrate counterion specificity for a set of common ions, Figure 2 shows the calculated potential of mean force between two like-charged macroions of diameter equal to $2 \mathrm{~nm}$ at center-to-center distance of $2.6 \mathrm{~nm}$, for solutions at ionic strength $0.125 \mathrm{M}$ for different salts. The center-to-center distance of 2.6 $\mathrm{nm}$ is chosen in the range where ion-specific effects are still sufficiently strong, while the uncertainties due to neglecting of molecular solvation (most significant in the macroion hydration shell) become less significant. As in previous calculations, results do not include the direct macroion-macroion dispersion (Hamaker) force.

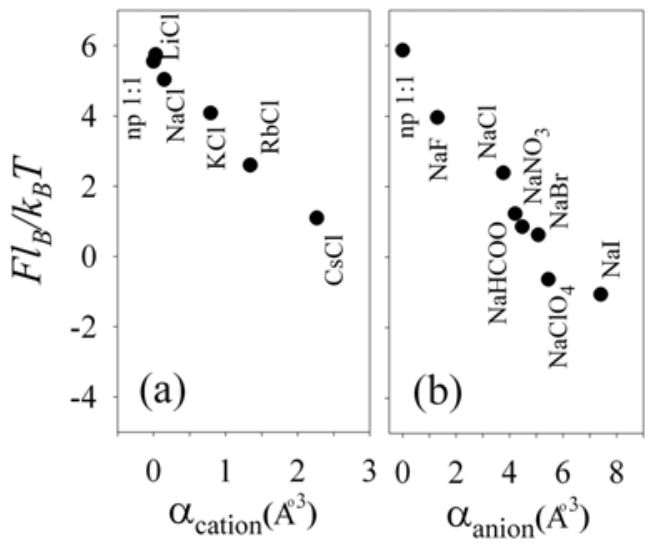

Fig. 2 - Effect of counterion identity on the potential of mean force between two macroions at a fixed distance $\left(r=1.3 \sigma_{m}\right)$ in a solution of ionic strength $0.125 \mathrm{M}$. In (a), the macroion net charge number is -20 , the counterions are cations. In (b), the macroion net charge number is +20 , the counterions are anions. Raising counterion polarizability, increases the attraction between two macroions (Tavares et al. 2004a).

The affinity of an anion for an anion-exchange resin depends on the particular resin, but the general trend (Gjerde et al. 1980) is given by the order $\mathrm{SCN}^{-}>\mathrm{I}^{-}>\mathrm{Br}^{-}>\mathrm{Cl}^{-}$. Also, Everett (1988) reported that the experimental effectiveness of monovalent cations in coagulating a negatively charged colloid usually varies in the order $\mathrm{Cs}^{+}>\mathrm{Rb}^{+}>\mathrm{K}^{+}>\mathrm{Na}^{+}>\mathrm{Li}^{+}$. The same sequence holds for ionic adsorption at surfaces, for ester hydrolysis, and for precipitation of albumin (Gjerde et al. 1980). These experimental 
results agree with the sequences shown in Figure 2 for anions and cations.

While our model relies on an approximate McMillan-Mayer representation of the solvent, the present results are qualitatively consistent with specific-salt effects observed experimentally by numerous investigators, starting with Hofmeister for aqueous proteins over 100 years ago.

\section{MODIFIED POISSON-BOLTZMANN EQUATION AND COMPARISON WITH OZ/HNC AND MC}

The distribution of ions at a charged surface is a fundamental problem of colloid and interface science. The ions are considered as point charges embedded in a continuum of a uniform dielectric constants. In the classical theory, the chemical potential, $\mu_{i}$, of the ion ' $i$ ' with charge, $e z_{i}$, is given by:

$$
\mu_{i}=\mu_{0 i}+k_{B} T \ln \left(c_{i}\right)+e z_{i} \phi,
$$

where, $\mu_{0 i}$ represents the standard chemical potential of the ion of species ' $i$ ', $k_{B}$ is the Boltzmann constant, $T$ is the temperature, $c_{i}$ stands for the ion concentration, $e$ is the elementary charge, $z_{i}$ is the ion valence and $\phi$ is the self-consistent electric potential. In thermal equilibrium, $\mu_{i}$ remains constant throughout the system. Consequently, each sort of ion ' $i$ ' obeys the Boltzmann distribution.

$$
c_{i}=c_{0 i} \exp \left(-\frac{z_{i} e \phi}{k_{B} T}\right)
$$

where, $c_{0 i}$ is the ion concentration at the bulk reservoir.

The classical theory accounts only for electrostatics and thermal motion and neglects several important effects such as dispersion forces, fluctuation, hydration, ion size effects and the water structure at the interface. Several extensions of the theory are aiming at including one of these contributions to the model in the framework of mean-field Poisson-Boltzmann theory.

The fundamental Poisson equation is used to self-consistently relate the electric potential $\phi$ to the net excess charge density at a position $x$ :

$$
\nabla \cdot\left(\varepsilon_{0} \varepsilon_{w}(\bar{x}) \nabla \phi(x)\right)+\rho(\bar{x})=0
$$

where, $\varepsilon_{0}=8.854 \times 10^{-12} C^{2} /(\mathrm{Jm})$ is the dielectric permittivity of vacuum, $\varepsilon_{w}$ is the dielectric constant of the solvent and $\rho(\bar{x})$ is the net charge density at a given position, defined as:

$$
\rho(\bar{x})=e \sum_{i} z_{i} c_{i}(\bar{x})
$$

The interaction between an ion and a surface is not only electrostatic; each ion experiences a further additive term that comes partly from dispersion forces, $U_{i}$. Then, we have a modified Boltzmann distribution of ions in the solution:

$$
c_{i}=c_{0 i} \exp \left(-\frac{\left[z_{i} e_{\phi}+U_{i}\right]}{k_{B} T}\right) .
$$

The combination of Boltzmann distribution and Poisson equation for net excess charge density leads to a nonlinear second-order differential equation for the electric potential $\phi$. Therefore, the Poisson-Boltzmann equation is written

$$
\nabla \cdot\left(\varepsilon_{0} \varepsilon_{w}(x) \nabla \phi(x)\right)+e \sum_{i} z_{i} c_{o i} \exp \left(-\frac{z_{i} e \phi(x)+U_{i}(x)}{k_{B} T}\right)=0 .
$$


A question that can be raised is whether or not ion size correlation and electrostatic correlation, not accounted for in MPBE, may influence the result. Solvent averaged Monte Carlo (MC) simulations were performed by Boström et al. (2005b) for ion distributions outside a single globular macroion in different salt solutions. The model we used included both electrostatic and NES interactions among ions and between ions and macroions.

Simulation results were compared with the predictions of the Ornstein-Zernike equation (OZ) with the hypernetted chain (HNC) closure approximation and the non-linear Poisson-Boltzmann equation in spherical coordinates, both extended to include Lifshitz NES potentials. We show in Figure 3 that there is good agreement among modified Poisson-Boltzmann theory, MC simulations, and HNC calculations when the counterions and co-ions are monovalent. There is also good agreement among the different approaches with divalent co-ions (not shown here) (Boström et al. 2005b). However, the results from MPBE cannot account for ion size correlation and neither for electrostatic correlation. These correlation effects become more important for divalent counterions, as shown in Figure 4. The reason is simply that the divalent counterions accumulate close to the colloid surface preventing, by size exclusion, co-ions to come close to the colloid surface. The co-ions form a second layer outside the first layer.

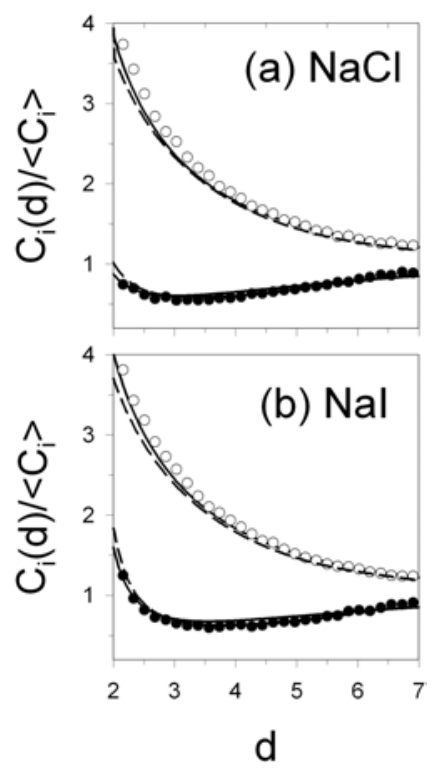

Fig. 3 - Concentration profiles near a macroion $\left(\sigma_{M}=30 \AA\right.$ and $\left.-20 \mathrm{e}^{0}\right)$ in a monovalent electrolyte solution of ionic strength $1.0 \mathrm{M}$ for $\mathrm{NaCl}$ (a) and $\mathrm{NaI}$ (b). Open circles represent counterion concentrations, and dark circles the coions. Solid lines are numerical solutions of the non-linear Poisson-Boltzmann equation and dashed lines are for the OZ-HNC integral equation (Boström et al. 2005b).

However, the general conclusion is that MPBE is, in many cases, a good approximation as compared to mean-field MC simulations or HNC calculations that account for ion size correlations. This motivates the success of the MPBE.

Other modified PB equations have been designed in the literature to incorporate better ion size correlation effects. In this matter, we refer to the series of papers by Lozada-Cassou and co-workers (JimenezAngeles et al. 2006, Messina et al. 2002). 


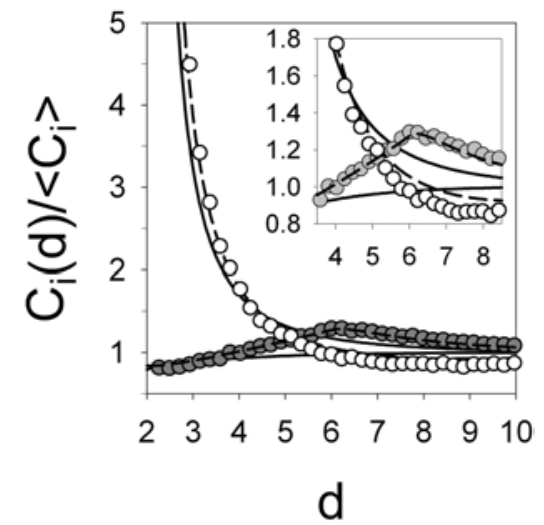

Fig. 4 - Concentration profiles near a macroion $\left(\sigma_{M}=30 \AA\right.$ and $\left.+20 \mathrm{e}^{0}\right)$ in solution of ionic strength $1.0 \mathrm{M}_{\text {for }} \mathrm{Na}_{2} \mathrm{SO}_{4}(\mathrm{Boström}$ et al. 2005b). Symbols refer to MC simulation results. Open circles represent counterion concentrations (sulfate) and gray circles the coions $\left(\mathrm{Na}^{+}\right)$. Solid lines are numerical solutions of the non-linear Poison-Boltzmann equation, and dashed lines are for the OZ-HNC integral equation. The inner figure shows that the HNC calculations for coion distributions coincide with the simulation results.

\section{USING MPB EQUATION TO UNDERSTAND REVERSAL OF HOFMEISTER SERIES}

We next consider two planar surfaces coated with lysozyme proteins, whose isoelectric point is $\mathrm{pI} \approx 11$. The purpose is to investigate if one can understand why the Hofmeister sequences can be totally different when the ions are counterions as compared to when they are co-ions. Assuming that we know the correct ion distributions, the double layer pressure between two planar plates (see section IV) a distance L apart can be written as (Lima et al. 2008a),

$$
P=k T \sum_{i}\left[c_{i}(L / 2)-c_{o, i}\right]-2 \sum_{i} \int_{x_{o}}^{L / 2} c_{i} \frac{d U_{i}}{d L} d x .
$$

In order to compute the change of surface charge density as a function of $\mathrm{pH}$, we have used charge regulated surfaces (Boström et al. 2005c). Details about charge regulation model and its implementation are given by Ninham and Parsegian (1970a) and Boström et al. (2005c).

In Figure 5 we show the sum of co-ion and counter-ion concentrations (normalized with bulk values) for $0.15 \mathrm{M} \mathrm{NaCl}$ and $0.15 \mathrm{M} \mathrm{NaSCN}$ at $\mathrm{pH} 4$ and $\mathrm{pH} 12$. It is clear from this figure that the first term in the double layer pressure (which is directly related to the concentration in the midpoint) gives rise to a reverse Hofmeister sequence at $\mathrm{pH} 4$, and a direct Hofmeister sequence at $\mathrm{pH} 12$. Below the isoelectric point, there is strong counter-ion adsorption of $\mathrm{SCN}^{-}$. This leads to a reduction in the total number of ions in the midplane between the plates and a reduced repulsion, as compared to the corresponding case for $\mathrm{Cl}^{-}$. Above the isoelectric point, there can be co-ion adsorption with $\mathrm{SCN}^{-}$. This leads to a higher concentration of counter-ions $\left(\mathrm{Na}^{+}\right)$between the two surfaces. While this co-ion adsorption leads to slightly less co-ions with thiocyanate, as compared with chloride, the dominant effect is that it increases the number of counter-ions between the plates. There is a strong clue that suggests that this is the main source of the different Hofmeister sequences observed when $\mathrm{pH}$ is below or above the isoelectric point.

The second term in Eq. (18) for the double layer pressure is related to the ionic dispersion interaction between the ions and the two plates. For attractive dispersion potentials, this term gives an attraction. At 


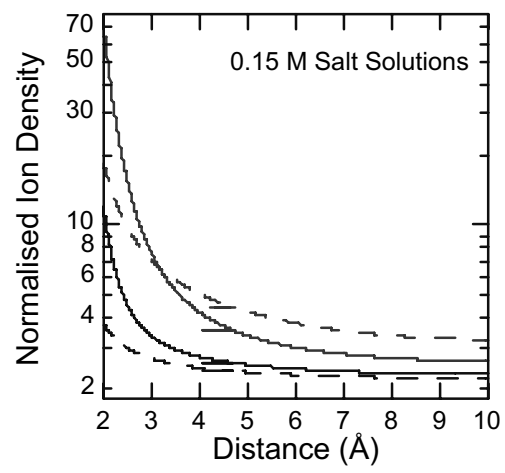

Fig. 5 - The ion density (anion+cation) normalized with the bulk values in between two charge regulated plates (that carry the same charge density and charge groups as hen-egg-white lysozyme, which is supposed to be uniformly smeared out on the surface) $20 \AA$ apart. The upper (lower) two curves are for $\mathrm{pH} 4$ (pH 12) and the solid (dashed) lines correspond to $0.15 \mathrm{M} \mathrm{NaSCN}(0.15 \mathrm{M}$ $\mathrm{NaCl})$ (Boström et al. 2005c).

least in the examples considered here, it increases with the magnitude of the dispersion potential. The two terms together give rise to a situation where the total pressure due to the salt follows a reverse Hofmeister sequence for $\mathrm{pH}<\mathrm{pI}$, and a direct Hofmeister sequence for $\mathrm{pH}>\mathrm{pI}$.

We are now ready to look in some detail at how the pressure for a fixed plate separation $(20 \AA)$ changes with $\mathrm{pH}$ as we cross the isoelectric point. The calculation was done for lysozyme coated plates $(\mathrm{pI} \approx 11.16)$ for different $0.15 \mathrm{M}$ salt solutions. However, the qualitative results would be valid for other proteins as well. In excellent agreement with experiments on a variety of proteins (with lysozyme the experiments could not be done above $\mathrm{pI}$ ), we see in Figure 6 that we obtain a reverse Hofmeister sequence for $\mathrm{pH}<\mathrm{pI}$, and a direct Hofmeister sequence for $\mathrm{pH}>\mathrm{pI}$. Close to the isoelectric point, where electrostatic effects are very small, one can even have attraction with very polarizable anions such as thiocyanate.

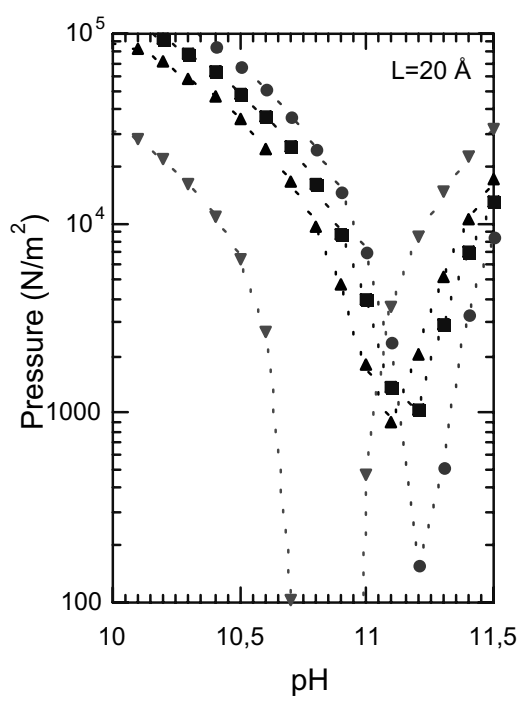

Fig. 6 - The double layer pressure in $0.15 \mathrm{M}$ salt solutions between two plates carrying the same charge density and charge groups as lysozyme proteins $20 \AA$ apart. We are in all examples excluding the direct van der Waals pressure between the two plates across a water solution. The different curves correspond to: electrostatics (circles); $\mathrm{NaCl}$ (squares); NaI (triangles pointing up); and NaSCN (triangles pointing down). (The lines are only there to aid the eye) (Boström et al. 2005c). 


\section{USING MPB EQUATION IN BISPHERICAL COORDINATES}

There is a considerable effort in the literature trying to calculate the mean force among proteins and among colloidal particles. In this context, the Poisson-Boltzmann (PB) equation can be a useful way because it is solved with reduced computational cost, compared with molecular simulations and integral equation theories, and also because it does not present numerical instabilities for large asymmetrical systems.

We use now the ion-specific MPB equation in bispherical coordinates. This Modified PoissonBoltzmann equation enables us to predict ion-specific properties of colloidal systems by studying the interactions between two spherical particles. Phase diagrams and osmotic second virial coefficients are presented for different salt solutions at different $\mathrm{pH}$ and ionic strengths, in agreement with the experimental observed Hofmeister effects.

The mean force between two proteins immersed in an electrolyte solution is a function of the electrostatic potential profile. We have implemented the finite volume method (Lima et al. 2007a) to solve the ion-specific Poisson-Boltzmann equation for two dissimilar colloidal particles immersed in an electrolyte solution, including sphere-sphere (Lima et al. 2007a, c) and plane-sphere geometries (Lima et al. 2007b). The method has been proved to be efficient and suitable for PB calculations. The PB equation in bispherical coordinates, considering ion-colloid van der Waals dispersion interactions, has successfully predicted the ion specificity commonly observed experimentally, as shown here in Figures $7 \mathrm{a}$ and $7 \mathrm{~b}$. We emphasize that our calculations are predictive, i.e., there are no adjustable parameters. In order to simulate the interaction between lysozyme particles, we use the charge regulation model (Ninham and Parsegian 1970a).

Figure 7a shows a comparison of the calculated osmotic second virial coefficient for lysozymelysozyme interactions in $\mathrm{NaCl}$ solution at $\mathrm{pH} 4.5$ and $298 \mathrm{~K}$ with experimental data.

Figure $7 \mathrm{~b}$ shows phase diagrams for two aqueous lysozyme solutions containing $\mathrm{NaCl}$ or $\mathrm{NaI}$ at 298K, calculated from first-order perturbation theory (Tavares et al. 2004c) using the potential of mean force obtained from PB equation plus Hamaker contribution. These calculations are in good agreement with recent experimental and theoretical results by Gögelein et al. (2008). For both cases, salt concentration is $0.3 \mathrm{M}$. Stable fluid-fluid (dashed line) and fluid-solid (solid lines) transitions appear for solutions containing NaI. However, in $0.3 \mathrm{M} \mathrm{NaCl}$, with its relatively small attractive potential, the fluid-fluid phase transition is metastable, i.e., at a fixed temperature smaller than the fluid-fluid critical point, the equilibrium conditions (isochemical potential and isopressure equations) give two possibilities for phase coexistence: solid-fluid and fluid-fluid. However, the chemical potentials obtained for solid-fluid calculations are smaller than those for fluid-fluid. Therefore, the solid-fluid equilibrium is stable when compared with fluid-fluid equilibrium. At all packing fractions, this system shows a stable fluid-solid phase transition. The metastable fluid-fluid transition for $\mathrm{NaCl}$ is represented by a dashed line. Symbols (squares) are fluid-fluid critical points.

Therefore, important thermodynamic properties related to protein aggregation, essential in biotechnology and pharmaceutical industries, can be obtained from the method shown here.

\section{ION-SURFACE POTENTIAL OF MEAN FORCE FROM SIMULATIONS}

Mean-field theories that include non-electrostatic interactions acting on ions near interfaces, as we have discussed so far, have been found to accommodate many experimentally observed ion specific effects. However, it is clear that this approach does not fully account for the liquid molecular structure and hydration 


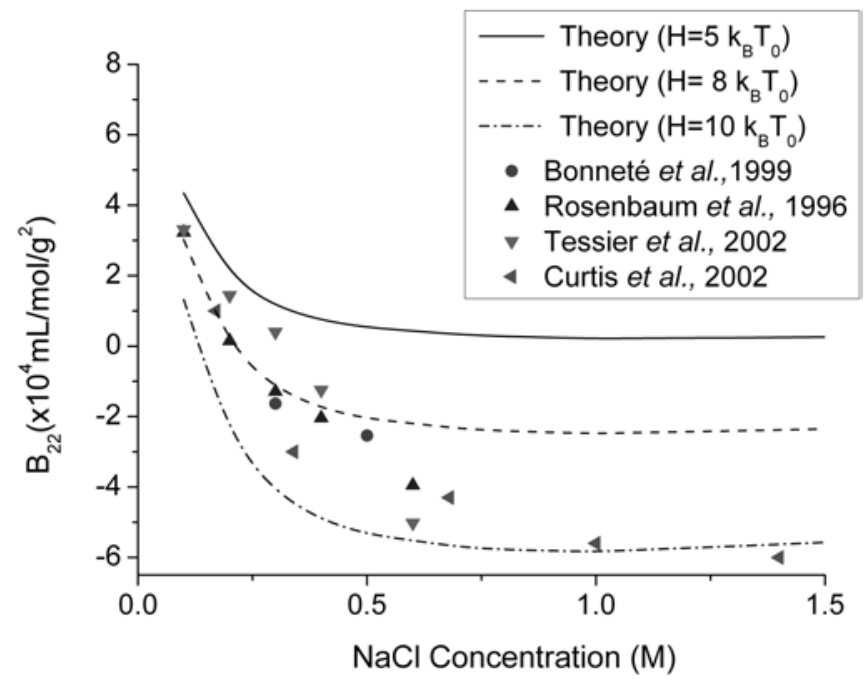

(a)

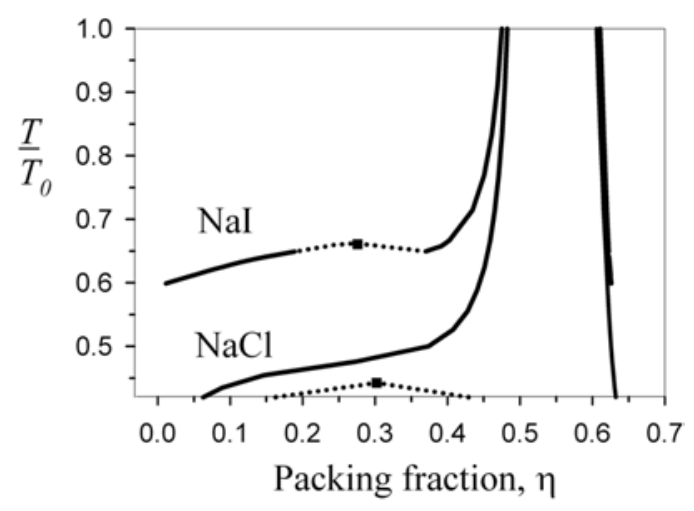

(b)

Fig. 7 - (a) Comparison of the theoretical second virial coefficient for lysozyme- lysozyme interactions (for three values of the Hamaker constant equal to $5 k_{B} T_{0}, 8 k_{B} T_{0}$ and $10 k_{B} T_{0}$ ) in $\mathrm{NaCl}$ solution at $\mathrm{pH} 4.5$ with experimental data; (b) Phase diagrams for two aqueous lysozyme solutions containing $\mathrm{NaCl}$ or NaI. For all cases, salt concentration is $0.3 \mathrm{M}$ and $T_{0}$ is $298 \mathrm{~K}$. Stable fluid-fluid (dashed line) and fluid-solid (solid lines) transitions appear for solutions containing $\mathrm{NaI}$. When the electrolyte is $\mathrm{NaCl}$, the stable transition is fluid-solid for all packing fractions. The metastable fluid-fluid transition for $\mathrm{NaCl}$ is represented by a dashed line. Symbols (squares) are fluid-fluid critical points. (Lima et al. 2007c, 2008c).

effects. This has recently been improved by using parameterized ionic potentials deduced from recent non-primitive model MD simulations in a generalized Poisson-Boltzmann equation (Horinek et al. 2008, Lima et al. 2008a, b, Horinek and Netz 2007). We investigated how ion distributions and double layer forces depend on the choice of the background salt. Here we consider ion distributions near a single charge neutral hydrophobic colloidal particle.

For a mixed electrolyte in spherical coordinates, the Poisson equation is,

$$
\frac{\varepsilon_{0}}{r^{2}} \frac{d}{d r}\left(r^{2} \varepsilon_{w}(r) \frac{d \phi}{d r}\right)=-e\left[c_{N a^{+}}(r)-c_{C l^{-}}(r)-c_{I^{-}}(r)\right],
$$

where $\phi$ is the self-consistent electrostatic potential. Using the modified Boltzmann distribution, the ion concentrations as functions of the radial distance $c_{i}(r)$ are given by Eq. (16). In Eq. (16), the NES potential $U_{i}$ experienced by the ions can be represented by the ionic potential of mean force (PMF) obtained from molecular simulation. This PMF receives contributions from different sources. Here we consider, as an illustrative example, ion distributions near a spherical gold nanoparticle covered with an uncharged hydrophobic self-assembled monolayer (SAM). Horinek and Netz (2007) recently performed molecular dynamics simulations that produced potential of mean force (PMF) acting on different ions near a SAM. The PMF obtained includes image-charge effects, van der Waals interactions among ion, water, and substrate and ion hydration. These PMFs are reproduced graphically in Figure 8 for $\mathrm{Na}^{+}, \mathrm{Cl}^{-}$, and $\mathrm{I}^{-}$. Since this PMF is taken from infinite dilution of electrolyte, it contains the unscreened image charge effect. Therefore, one has to be cautious when it is applied at finite electrolyte concentration. The unscreened self-image term gives a too long-ranged influence on the density profiles. 


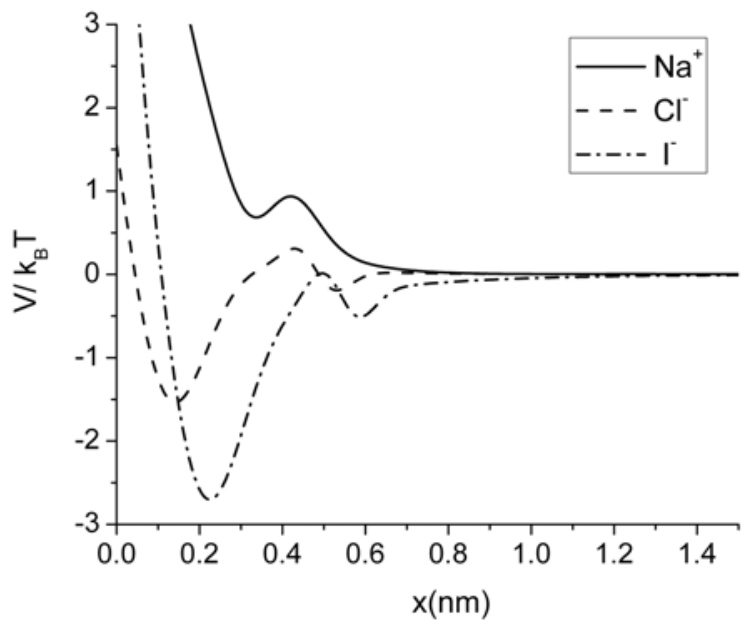

Fig. 8 - Potential of mean force for different ions near the SAM-water interface from molecular simulation (Horinek and Netz 2007).

The inhomogeneous dielectric constant profile $\varepsilon_{w}(r)$ used here has little influence on the final result reported here and is given by Lima et al. (2008a). The boundary conditions come from the assumption of global charge neutrality and that we have no charges at infinity. The electric field at the charge neutral colloidal particle surface is:

$$
\left.\left(r^{2} \frac{d \phi}{d r}\right)\right|_{r_{c}}=0
$$

Solving Eqs. (16), (19) and (20) combined with PMFs reproduced in Figure 8, we calculated the electrostatic potential profile and the ion distributions (local concentrations). The ion distributions for the three ions are shown in Figure 9 for $0.2 \mathrm{M}$ total salt concentration. We observe an enhanced surface anion concentration due to ion specific PMF that is largest for iodide ions. In Figure 10 we consider a system with the same total salt concentration, but with pure $\mathrm{NaCl}$. In this case, we observe a physisorption of chloride ions. Comparing Figures 9 and 10, we notice that the physisorption of chloride is slightly reduced by the presence of iodide ions competing for the surface.

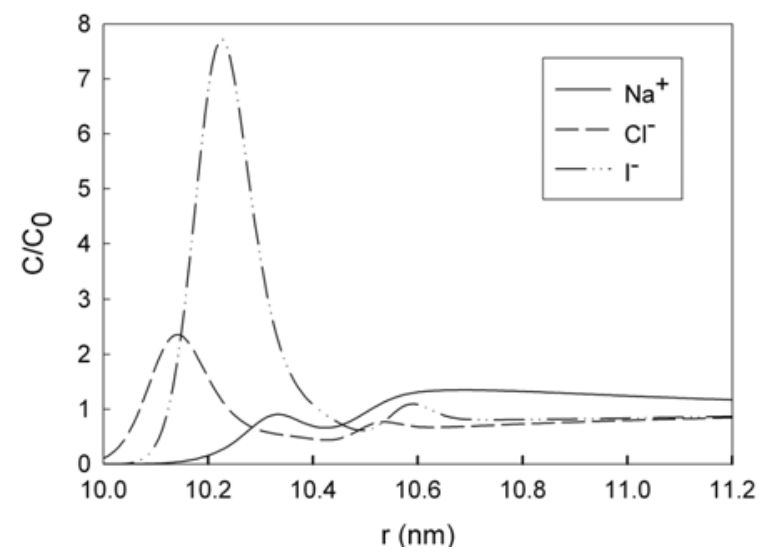

Fig. 9 - Concentration profile of anions and cations (mixed salt with equal concentrations of $\mathrm{NaCl}$ and $\mathrm{NaI}$ ) outside of an uncharged hydrophobic colloidal particle with radius $10 \mathrm{~nm}$ at a total bulk concentration $\mathrm{C}_{0}=0.2 \mathrm{M}$. 


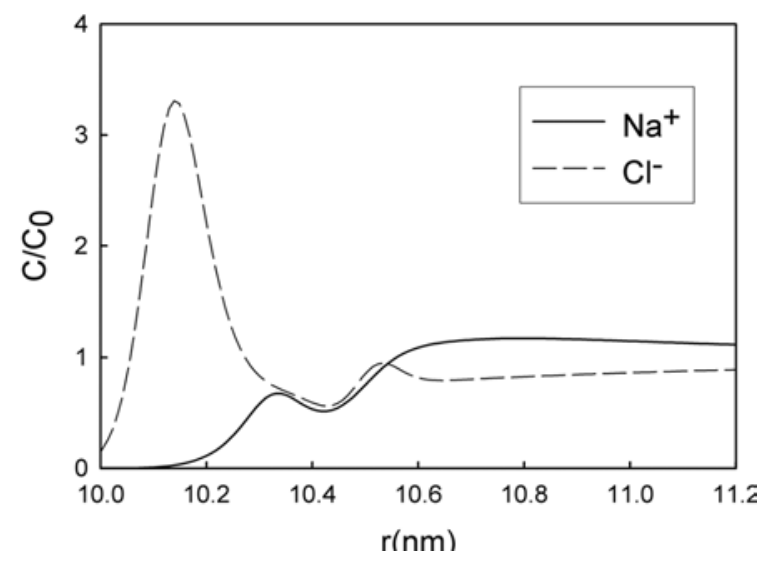

Fig. 10 - Concentration profile of $\mathrm{Cl}^{-}$anions and $\mathrm{Na}^{+}$cations outside of an uncharged hydrophobic colloidal particle with radius $10 \mathrm{~nm}$ at a bulk concentration $\mathrm{C}_{0}=0.2 \mathrm{M}$ of $\mathrm{NaCl}$.

We have shown that there can be highly specific ion adsorption to colloidal particles. As shown by the beautiful experiment performed by Lo Nostro and co-workers (Lagi et al. 2007), this may lead to anion specific concentrations in dense phases. We propose that, in a system containing two phases (each phase with finite volume), ions can be pushed into the phase with larger surface areas (dense phase) due to ion specific adsorption. The same binding mechanism should also be present in any system where ions can permeate inside a region with large surface adsorption area, such as lipids, proteins and/or polymers, and bind to the surfaces in an ion specific way. This result should be of relevance for colloid science and cell physiology.

\section{CONCLUSIONS}

The mean force between two colloidal particles in salt solutions has been calculated including both electrostatic interactions and salt-specific NES potentials among small ions and between small ions and macroions. Monte Carlo simulations and Modified Poisson-Boltzmann equation have been used to determine effects of salt identity on the potential of mean force between two proteins or colloidal particles. While our model relies on a solvent averaged model, the present results are qualitatively consistent with specific-salt effects observed experimentally by numerous investigators, starting with Hofmeister for aqueous proteins over 100 years ago. Our calculations emphasize the importance of NES potentials between ions and macroions. These specific forces, along with effects related to molecular solvation, may provide a key toward understanding salt-type effects as observed for colloidal and protein solutions. Recently theories using ionic dispersion potentials have been improved by using ion-surface PMF from simulations that include explicit water molecules. We believe that we are now close to qualitative understanding of the Hofmeister effect.

\section{ACKNOWLEDGMENTS}

We thank Barry W. Ninham, John M. Prausnitz, Dusan Bratko, Dominik Horinek, Roland R. Netz, Roland Kjellander, and Werner Kunz for fruitful discussions, comments and collaboration in several articles presented here. We also thank the Brazilian Agencies, Coordenação de Aperfeiçoamento de Pessoal de Nível Superior (CAPES), Fundação Carlos Chagas Filho de Amparo à Pesquisa do Estado do Rio de Janeiro 
(FAPERJ), Conselho Nacional de Desenvolvimento Científico e Tecnológico (CNPq), and the Swedish Research Council, the German Arbeitsgemeinschaft industrieller Forschungvereinigungen Otto von Guericke e.V. (AiF), for financial support.

\section{RESUMO}

Interações íon-específicas (dependentes do tipo de íon presente em solução) entre duas partículas coloidais são calculadas usando a equação de Poisson-Boltzmann (PB) modificada e simulações de Monte Carlo (MC). As equações de PB apresentam bons resultados de perfis de concentração nas proximidades de um macro-íon, principalmente para soluções salinas contendo íons monovalentes. Estas equações incluem não só interações eletrostáticas, mas também potenciais de dispersão, que têm origem nas polarizabilidades de íons e proteínas, permitindo a predição de propriedades íon-específicas de sistemas coloidais. Os resultados obtidos a partir da equação de PB modificada são comparados com outros obtidos por simulação de $\mathrm{MC}$ e por equações integrais. Diagramas de fase e o segundo coeficiente de virial são obtidos para diferentes sais e diferentes valores de $\mathrm{pH}$ e força iônica, em concordância com efeitos de Hofmeister observados experimentalmente. Interações efetivas obtidas por dinâmica molecular entre cada íon e uma superfície hidrofóbica foram incluídas na equação de PB, a fim de considerar a estrutura da água e efeitos de hidratação. O método mostrou-se eficiente e adequado para descrever fenômenos onde a estrutura da água nas proximidades da interface desempenha papel essencial. Propriedades termodinâmicas importantes, relacionadas com a agregação de proteínas, essenciais em biotecnologia e indústrias farmacêuticas, podem ser obtidas pelo método aqui apresentado.

Palavras-chave: estabilidade de colóides, série de Hofmeister, potencial de campo médio, proteínas.

\section{REFERENCES}

Boström M AND Ninham BW. 2004. Contributions from dispersion and Born self-free energies to the solvation energies of salt solutions. J Phys Chem B 108: 12593-12595.

Boström M AND NinHAM BW. 2005. Energy of an ion crossing a low dielectric membrane: the role of dispersion self-free energy. Biophys Chem 114: 95-101.

Boström M, Williams D And Ninham BW. 2001a. Specific ion effects: Why DLVO theory fails for biology and colloid systems. Phys Rev Lett 87: 168103.

Boström M, Williams DRM And Ninham BW. 2001b. Surface tension of electrolytes: specific ion effects explained by dispersion forces. Langmuir 17: 4475-4478.

Boström M, Kunz W And Ninham BW. 2005a. Hofmeister effects in surface tension of aqueous electrolyte solution. Langmuir 21: 2619-2623.

Boström M, Tavares FW, Bratko D and Ninham BW. 2005b. Specific ion effects in solutions of globular proteins: Comparison between analytical models and simulation. J Phys Chem B 109: 24489-24494.

Boström M, Tavares FW, Finet S, Skouri-Panet F, TARdieu A and Ninham BW. 2005c. Why Forces between Proteins follow different Hofmeister series for $\mathrm{pH}$ above and below pI. Biophys Chem 117: 217-224.

Boström M, Tavares FW, Ninham BW and Prausnitz JM. 2006. Effect of salt identity on the phase diagram for globular protein in aqueous electrolyte solution. J Phys Chem B 110: 24757-24760.

Carbonnaux C, Riés-Kautt MM and Ducruix A. 1995. Relative effectiveness of various anions on solubility of acidic hypoderma-lineatum collagenase at pH 7.2. Protein Sci 5: 2123-2128. 
COLLINS KD. 2004. Ions from the Hofmeister series and osmolytes: effects on proteins in solution and in the crystallization process. Methods 34: 300-311.

Collins KD and Washabaugh MW. 1985. The Hofmeister effect and the behavior of water at interfaces. Quart Rev Biophys 18: 323-422.

Craig V, Ninham BW and Pashley R. 1993. Effects of electrolytes on bubble coalescence. Nature 364: $317-319$.

Curtis Ra, Ulrich J, Montaser A, Prausnitz JM and Blanch HW. 2002. Protein-protein interactions in concentrated electrolyte solutions - Hofmeister-series effects. Biotechnol Bioeng 79: 367-380.

EverETt DH. 1988, Basic principles of colloid science. London: The Royal Society of Chemistry.

FRANKS GJ. 2002. Zeta potentials and yield stresses of silica suspensions in concentrated monovalent electrolytes: Isoelectric point shift and additional attraction. Colloid Int Sci 249: 44-51.

GJERDE DT, SCHMUCKLER G AND FRITZ JS. 1980. Anion chromatography with low-conductivity eluents II. J Chromatogr 187: 35-45.

Gögelein C, Nägele G, Tuinier R, Gibaud T, Stradner A and Schurtenberger P. 2008. A simple patchy colloid model for the phase behavior of lysozyme dispersions. J Chem Phys 129: 085102.

Horinek D AND Netz RR. 2007. Specific ion adsorption at hydrophobic solid surfaces. Phys Rev Lett 99: 226104.

Horinek D, Serr A, Bonthius D, Boström M, Kunz W and Netz RR. 2008. Molecular hydrophobic attraction and ion-specific effects studied by molecular dynamics. Langmuir 24: 1271-1283.

ISRAELACHVILI JN. 1992. Intermolecular and surface forces. London: Academic Press.

Jimenez-Angeles F, OdRiozola G AND LOzADA-CASSOU M. 2006. Electrolyte distribution around two like-charged rods: Their effective attractive interaction and angular dependent charge reversal. J Chem Phys 124: 134902.

Kim H-K, Tuite E, Norden B And Ninham BW. 2001. Co-ion dependence of DNA nuclease activity suggests hydrophobic cavitation as a potential source of activation energy. Eur Phys J E 4: 411-417.

Kunz W, Henle J And Ninham BW. 2004a. 'Zur Lehre von der Wirkung der Salze' (about the science of the effect of salts): Franz Hofmeister's historical papers. Curr Op Colloid Int Sci 9: 19-37.

Kunz W, Lo Nostro P And Ninham BW. 2004b. The present state of affairs with Hofmeister effects. Curr Op Colloid Int Sci 9: 1-18.

Lagi M, Lo Nostro P, Fratini E, Ninham BW and Baglioni P. 2007. Insights into Hofmeister mechanisms: anion and degassing effects on the cloud point of dioctanoylphosphatidycholine/water systems. J Phys Chem B 111: 589-597.

Lima ERA, TAVARES FW AND BisCAIA JR EC. 2007a. Finite volume solution of the modified Poisson-Boltzmann equation for two colloidal particles. Phys Chem Chem Phys 9: 3174-3180.

Lima ERA, Biscaia JR EC, Boström M And TAVARes FW. 2007b. Ion specific forces between a nanoprobe and a charged surface. Langmuir 23: 7456-7458.

Lima ERA, Biscaia Jr EC, Boström M, Tavares FW and Prausnitz JM. 2007c. Osmotic second virial coefficients and phase diagrams for aqueous proteins from a much improved PB equation. J Phys Chem C 111: $16055-16059$. 
Lima ERA, Horinek D, Netz RR, Biscaia EC, Tavares FW, Kunz W and Boström M. 2008a. Specific ion adsorption and surface forces in colloid science. J Phys Chem B 112: 1580-1585.

Lima ERA, Boström M, Horinek D, Biscaia EC, Kunz W and Tavares FW. 2008b. Co-ion and ion competition effects: Ion distributions close to a hydrophobic solid surface in mixed electrolyte solutions. Langmuir 24: 3944-3948.

Lima ERA, Biscaia Jr EC, Boström M, Tavares FW and Prausnitz JM. 2008c. Osmotic second virial coefficients and phase diagrams for aqueous proteins from a much improved PB equation. Additions and Corrections. J Phys Chem C 112: 8741.

Lo Nostro P, Lo nostro A, Ninham BW, Pesavento G, Fratoni L and Baglioni P. 2004. Hofmeister specific ion effects in two biological systems. Curr Op Colloid Int Sci 9: 97-101.

Messina R, GonzÁlez-Tovar E, Lozada-Cassou M and Holm C. 2002. Overcharging: The crucial role of excluded volume. Europhys Lett 60: 383.

Ninham BW And PARSegian VA. 1970a. Van der Waals forces: special characteristics in lipid water systems and a general method of calculation based on the Lifshitz Theory. Biophys J 10: 646-663.

Ninham BW and Parsegian VA. 1970b. Van der Waals forces across triple layers films. J Chem Phys 52: $4578-4587$.

Ninham BW And YAminsky V. 1997. Ion binding and ion specificity: the Hofmeister effect and Onsager and Lifshitz theories. Langmuir 13: 2097-2108.

PaShley R And Ninham BW. 1987. Double-layer forces in ionic micellar solutions. J Phys Chem 91: 2902-2904.

Ries-KAUTT MM AND DUCRUIX AF. 1989. Relative effectiveness of various ions on solubility and crystal growth of lysozyme. J Biol Chem 264: 745-748.

Tavares FW, Bratko D, Blanch HW and Prausnitz JM. 2004a. Ion-specific effects in the colloid-colloid or protein-protein potential of mean force: role of salt-macroion van der Waals interactions. J Phys Chem B 108: 9228-9235.

Tavares FW, Bratko D And Prausnitz JM. 2004b. The role of salt-macroion van der Waals interactions in the colloid-colloid potential of mean force. Curr Op Colloid Int Sci 9: 81-86.

Tavares FW, Bratko D, Striolo A, Blanch HW and Prausnitz JM. 2004c. Phase behavior of aqueous solutions containing dipolar proteins from second-order perturbation theory. J Chem Phys 120: 9859-9869.

Vogel R. 2004. Influence of salts on rhodopsin photoproduct equilibria and protein stability. Curr Op Colloid Int Sci 9: 133-138.

Von HIPPer AR. 1958. Dielectric materials and applications. J Wiley \& Sons, New York, USA.

Wu JZ, Bratko D and Prausnitz JM. 1998. Interaction between like-charged colloidal spheres in electrolyte solutions. Proc Natl Acad Sci USA 95: 15169-15172.

Wu JZ, Bratko D, Blanch HW and Prausnitz JM. 1999. Monte Carlo simulation from the potential of mean force between ionic colloids in solutions of asymmetric salts. J Chem Phys 111: 7084-7094. 